\title{
Binding of Monovalent and Bivalent Ligands by Transthyretin Causes Different Short- and Long-Distance Conformational Changes
}

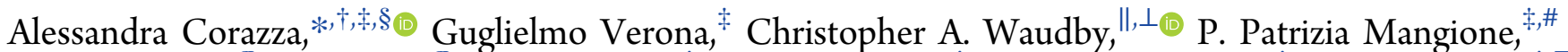
Ryan Bingham, ${ }^{\text {,I }}$ Iain Uings, ${ }^{\text {, }}$ Diana Canetti, ${ }^{\ddagger}$ Paola Nocerino, ${ }^{\ddagger}$ Graham W. Taylor, ${ }^{\ddagger}$ Mark B. Pepys, ${ }^{\ddagger, \nabla}$ John Christodoulou, ${ }^{\|, \perp}$ and Vittorio Bellotti*, ${ }^{*, \ldots}$

${ }^{\dagger}$ Department of Medicine (DAME), University of Udine, Udine 33100, Italy

${ }^{\ddagger}$ Wolfson Drug Discovery Unit, Centre for Amyloidosis and Acute Phase Proteins, Division of Medicine, University College London, London NW3 2PF, U.K.

${ }^{\S}$ Istituto Nazionale Biostrutture e Biosistemi, Roma 00136, Italy

"Institute of Structural and Molecular Biology, University College London, London WC1E 6BT, U.K.

${ }^{\perp}$ Institute of Structural and Molecular Biology, Birkbeck College, London WC1E 7HX, U.K.

\#Department of Molecular Medicine, Institute of Biochemistry, University of Pavia, Pavia 27100, Italy

${ }^{\mathbb{I}}$ GSK Medicines Research Centre, Stevenage, Hertfordshire SG1 2NY, U.K.

${ }^{\nabla}$ National Amyloidosis Centre, University College London and Royal Free Hospital, London NW3 2PF, U.K.

\section{Supporting Information}

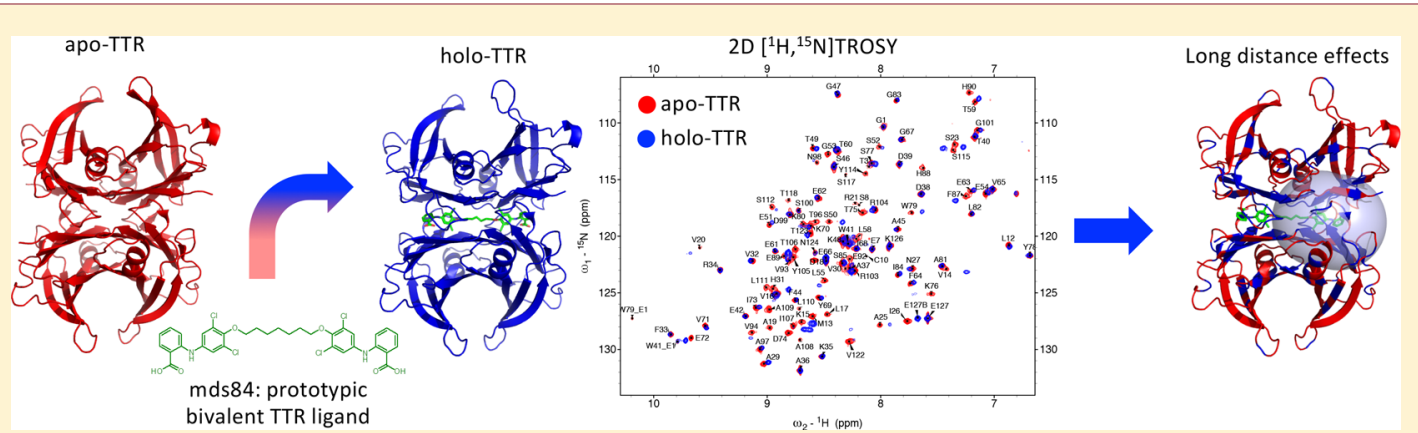

ABSTRACT: The wild type protein, transthyretin (TTR), and over 120 genetic TTR variants are amyloidogenic and cause, respectively, sporadic and hereditary systemic TTR amyloidosis. The homotetrameric TTR contains two identical thyroxine binding pockets, occupation of which by specific ligands can inhibit TTR amyloidogenesis in vitro. Ligand binding stabilizes the tetramer, inhibiting its proteolytic cleavage and its dissociation. Here, we show with solution-state NMR that ligand binding induces long-distance conformational changes in the TTR that have not previously been detected by X-ray crystallography, consistently with the inhibition of the cleavage of the DE loop. The NMR findings, coupled with surface plasmon resonance measurements, have identified dynamic exchange processes underlying the negative cooperativity of binding of "monovalent" ligand tafamidis. In contrast, mds84, our prototypic "bivalent" ligand, which is a more potent stabilizer of TTR in vitro that occupies both thyroxine pockets and the intramolecular channel between them, has greater structural effects.

\section{INTRODUCTION}

The normal wild type plasma protein, transthyretin (TTR) is inherently amyloidogenic, forming pathogenic extracellular amyloid deposits, most notably in the heart and predominantly in elderly men. In addition, there are over 120 different TTR gene mutations ${ }^{1}$ that cause autosomal dominant hereditary systemic TTR amyloidosis, affecting about 10000 individuals worldwide. ${ }^{2}$ Crystallographic studies, pioneered by Blake \& Oatley in $1977,{ }^{3}$ showed the noncovalent, homotetrameric structure of TTR (a dimer of dimers) and the presence of two identical binding sites located in a channel formed by the dimer-dimer interface and crossing the protein molecule. The first drug licensed for treatment of TTR amyloidosis, tafamidis, ${ }^{4}$ was devised as a specific ligand for TTR and developed on the basis of its capacity to inhibit aggregation of TTR at low $\mathrm{pH}$ in vitro. ${ }^{5}$ Other small molecules with this property have been extensively investigated ${ }^{6}$ but all share the characteristic of being "monovalent", that is, each ligand molecule only occupies a single thyroxine binding pocket in a single native tetrameric TTR molecule. In contrast, we devised "bivalent" TTR ligand compounds that are avidly, indeed pseudo-irreversibly, bound by native TTR under physiological conditions, occupying both binding pockets simultaneously and also the intramolecular

Received: June 29, 2019

Published: August 8, 2019 
A<smiles>O=C(O)c1ccc2nc(-c3cc(Cl)cc(Cl)c3)oc2c1</smiles>

B

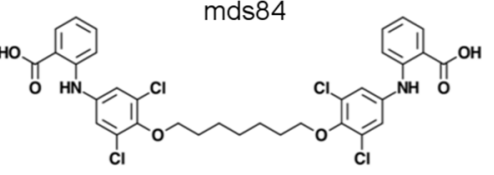

D

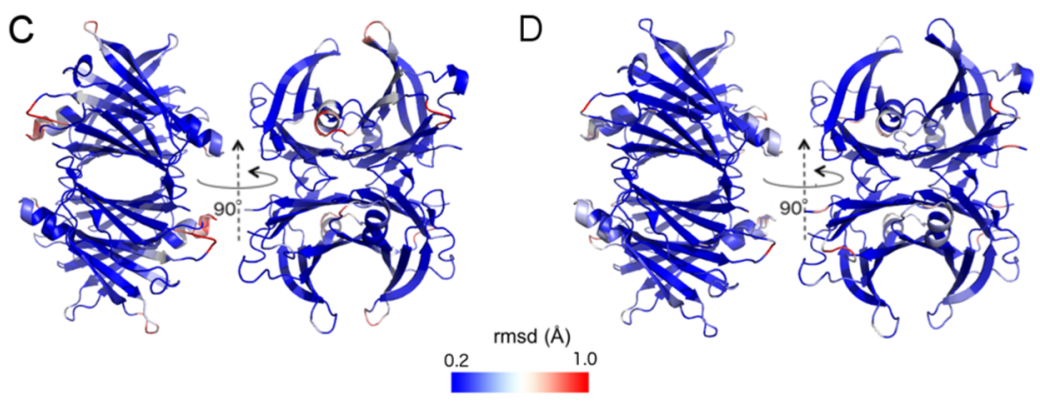

$2^{\operatorname{rmsd}(\AA)}$
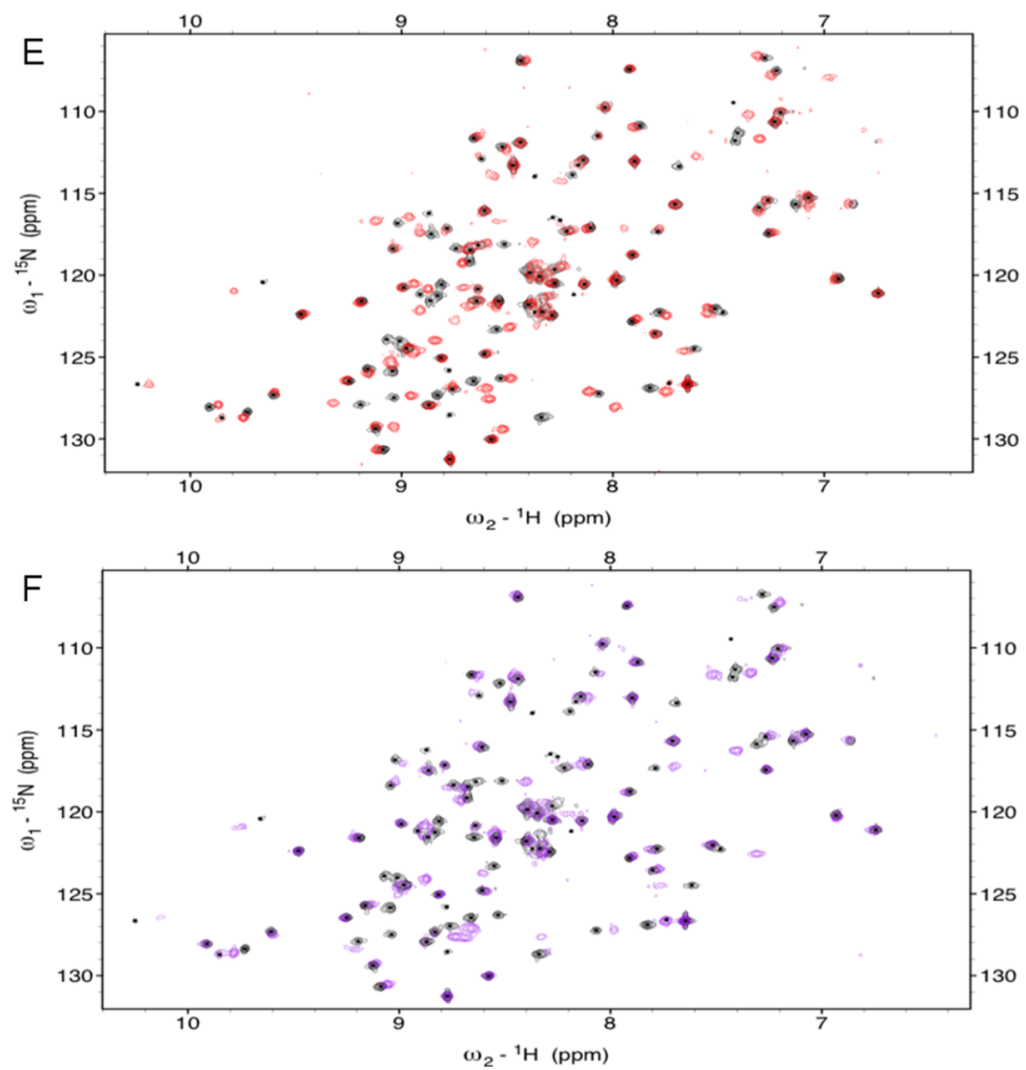

Figure 1. Effects of tafamidis and mds 84 binding by TTR shown by X-ray crystal structures and NMR spectra. (A,B) Chemical structures of tafamidis and mds84. (C,D) X-ray crystallographic structures of TTR-tafamidis (C, pdb 3TCT) and TTR-mds84 complexes (D, pdb 3IPE) in which the heavy atom rmsd difference from the apo-TTR form ( $\mathrm{pdb} 5 \mathrm{CN} 3$ ) is highlighted using a color gradient from minimal (blue) to maximal (red) differences. The average heavy atom rmsd values are 0.844 and 0.771 A in the presence of tafamidis and mds84, respectively. (E,F) Overlay of the $2 \mathrm{D}\left[{ }^{1} \mathrm{H},{ }^{15} \mathrm{~N}\right] \mathrm{TROSY}$ spectra, acquired at $800 \mathrm{MHz}$, of apo-TTR (black) and holo-TTR in the presence of tafamidis at 2:1 ratio (E, red) and mds84 at 1:1 ratio (F, purple).

channel that connects them. ${ }^{7}$ These compounds are much more potent inhibitors of acid-mediated TTR denaturation and aggregation in vitro. ${ }^{7}$ Crucially, the bivalent ligands are also more effective inhibitors of the physiologically relevant mechanism of TTR amyloid fibrillogenesis that involves mechanical forces and the specific, catalytic, proteolytic cleavage of TTR at $\mathrm{K} 48,{ }^{8-10}$ a process that is apparently mediated by plasmin in vivo. ${ }^{11}$

More recent high-resolution X-ray studies have shed light on the interaction between specific ligands and TTR and have informed chemical modifications to improve the binding properties of new ligands. ${ }^{12}$ However, a comparative analysis of crystallographic structures in the presence of ligands (holoTTR) or in their absence (apo-TTR) failed to identify significant differences in the protein structure. ${ }^{13}$ In contrast, our recent work on limited proteolysis of TTR suggests that, upon binding, changes on TTR conformation and/or dynamics might occur, even far from the site of interaction, particularly at loop $\mathrm{CD}$, where the proteolytic cleavage of the K48-T49 peptide bond occurs. ${ }^{9}$ While maximum resistance to proteolytic 
mds84:TTR

$0: 1$

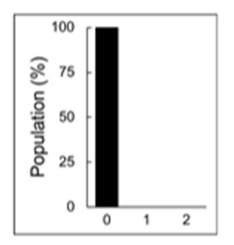

$0.6: 1$

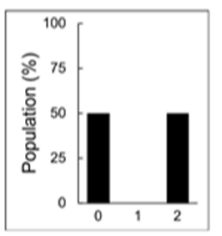

$1: 1$

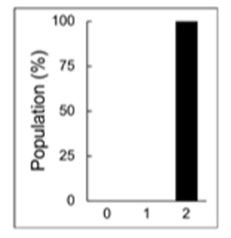

A
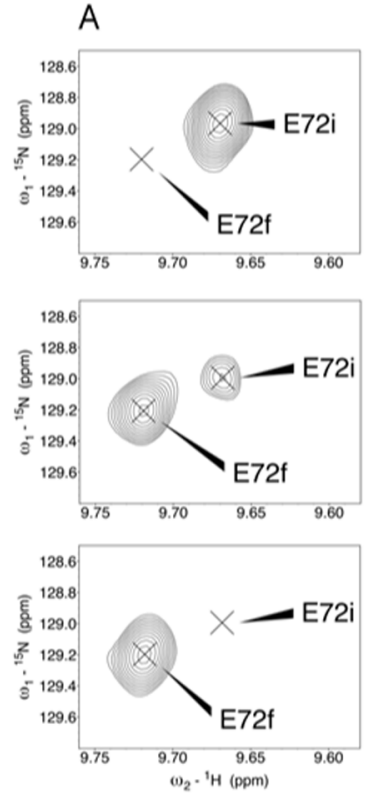

B
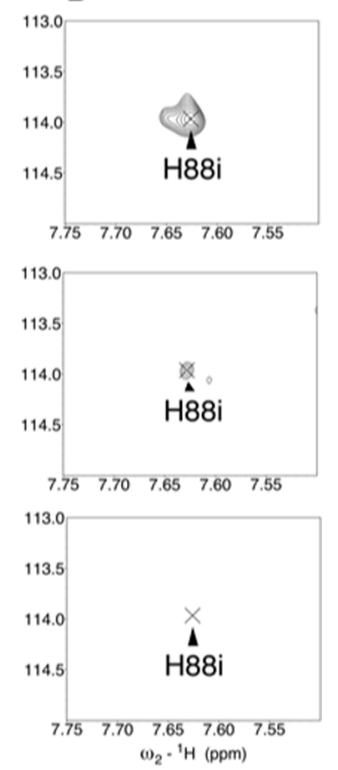

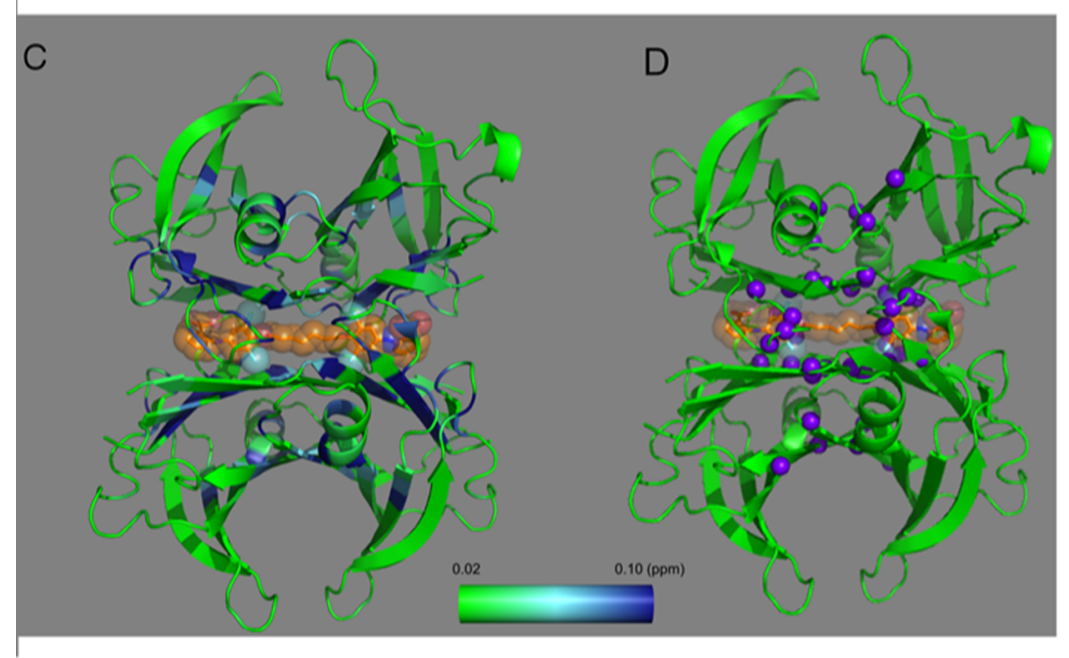

Figure 2. TTR binds the symmetrical bivalent ligand, mds84, in a slow exchange regime. The histograms represent the abundance of the TTR populations with 0,1 , and 2 binding sites occupied at molar mds84/TTR ratios of $0,0.5$, and 1 , based on the binding data of the literature. (A) E72 illustrating the behavior of most of the shifting peaks, 29 of 43, upon binding of mds84, according to a two-state model. The initial and final positions of the peaks are designated by $i$ and $f$, respectively, in the absence of ligand and with full occupancy of the two binding sites. (B) H88, a representative of the 14 peaks whose bound form is not observed, namely, L17, A19, R21, G22, L58, H88, E92, T96, A108, A109, L110, Y114, S117, and T118. (C) Combined chemical shift variations illustrated in a green-light blue-blue gradient color according to the scale shown, on the structure of the 1:1 TTR complex with mds84 (PDB: 3IPE). (D) Peaks observed with intermediate exchange regime shown as purple spheres.

cleavage of the holo-state of TTR, and thus protection against amyloidogenesis, is obtained when both sites are occupied by ligands, ${ }^{10}$ the known negative cooperativity of binding of the majority of TTR ligands ${ }^{14}$ militates strongly against this. Monovalent ligands such as tafamidis are bound by TTR, as is thyroxine, with negative cooperativity, with dissociation constants $K_{\mathrm{d} 1}$ and $K_{\mathrm{d} 2}$ of $\sim 2$ and $\sim 200 \mathrm{nM}$, respectively. ${ }^{4}$

A recent study demonstrated that, in contrast to $\mathrm{X}$-ray crystallography, NMR spectroscopy is able to detect changes in the solution structure of TTR caused by pathogenic amino acid mutations. ${ }^{15}$ Furthermore, it was found that conformational changes associated with different mutations were not restricted to the immediate proximity of the mutation site but included remote allosteric effects. Here, we used NMR to probe two prototypic TTR ligands: the monovalent ligand, tafamidis (Figure $1 \mathrm{~A})^{4}$ and the symmetrical bivalent compound, mds 84 (Figure 1B). ${ }^{7}$ We demonstrate that the unique simultaneous occupancy of both binding sites and the inner TTR channel by the bivalent ligand, in addition to short range effects, has major allosteric structural effects.

\section{RESULTS}

NMR Titrations Illuminate the Mechanism of Negative Cooperativity of TTR Ligand Binding. More than 25 very high-resolution X-ray structures have been reported of free wild type TTR as well as the complex of TTR bound to tafamidis and mds84, respectively. Figure S1 shows the TTR dimer with the labeling for each of the eight strands. The X-ray crystallographic 
tafamidis:TTR

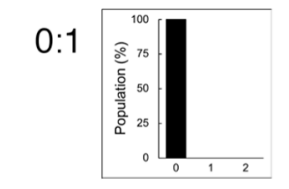

$0.5: 1$

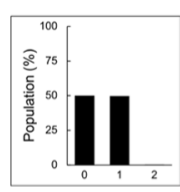

1.0:1

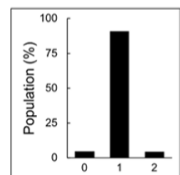

$1.5: 1$

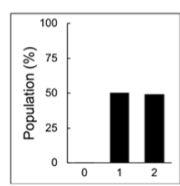

2.0:1

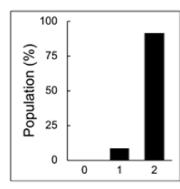

A B
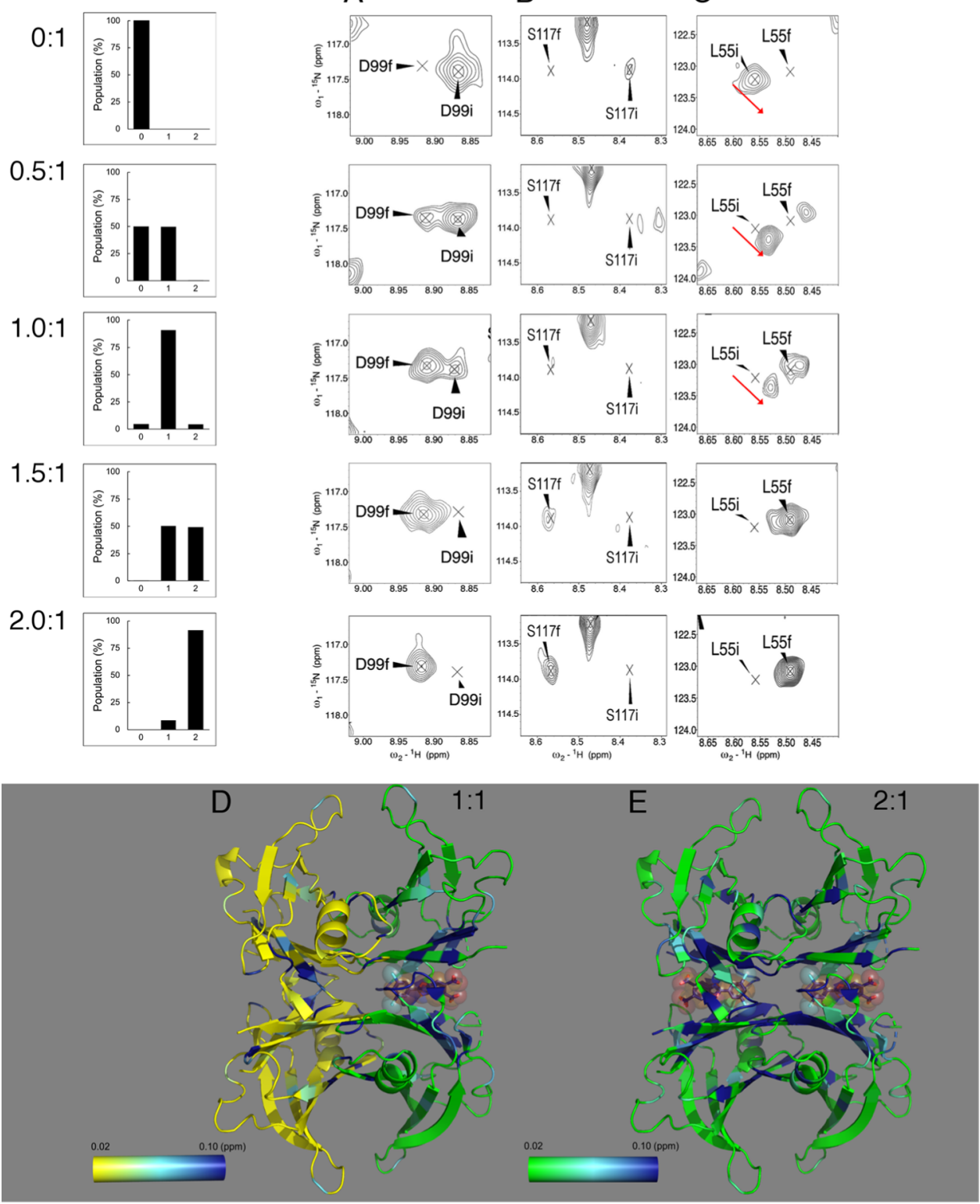

Figure 3. TTR binding of the monovalent ligand, tafamidis, and negative cooperativity. The histograms illustrate the populations with 0,1 , and 2 binding sites occupied by tafamidis at molar tafamidis/TTR ratios $0,0.5,1,1.5$, and 2 . Behavior of three prototypic peaks during the titration with tafamidis is illustrated. (A) D99, that exemplifies 10 residues, following a two-state model; (B) S117, that exemplifies 6 residues, showing an intermediate exchange, and (C) L55 exemplifying the majority of shifting peaks, 35 of 51, whose bound form not only decreases in intensity upon tafamidis binding, but also shifts, as indicated by the red arrow. The initial and final positions of the peaks, designated by $i$ and $f$, are, respectively, in the absence of ligand and with full occupancy of the two binding sites. (D) Only one of the two binding sites occupied at a 1:1 tafamidis/TTR ratio. The two opposite chains of TTR not bound to tafamidis are colored with a yellow-light blue-blue gradient, and the chains involved in the binding with a green-light blue-blue gradient, each according to their CSPs variations with the respective scale shown. (E) Variations of the combined chemical shift for holo-TTR saturated with two-fold molar excess of tafamidis are illustrated by a green-light blue-blue gradient according to the scale shown (PDB: 3ТСT).

structures of the apo- and holo-forms, with each of these different ligands, are almost identical. The heavy atom root mean square deviation (rmsd) is $<1 \AA$ (Figure 1C,D), although the holo-forms with both tafamidis and mds 84 have a marginally higher rmsd for the C-terminal end of helix 1 and for loops BC and FG than for the rest of the protein. The capacity of these ligands to inhibit TTR amyloid formation in vitro, ${ }^{9}$ by reducing the extent of proteolytic cleavage of the K48-T49 peptide bond, therefore suggests that ligand binding may produce allosteric modifications in TTR that may be revealed by solution studies despite not being detected in the crystal structures. The NMR backbone chemical shift assignment of wild type TTR has previously been reported under non-physiological $\mathrm{pH}$ and temperature conditions ${ }^{16}$ and a new assignment at neutral $\mathrm{pH}$ has recently been deposited in the bmrb databank. ${ }^{17}$

Here, we used TROSY multidimensional experiments ${ }^{18,19}$ to determine a de novo assignment for our TTR preparation, at 310 $\mathrm{K}$ and $\mathrm{pH}$ values of 7.0 and 7.4. At $\mathrm{pH} 7.4$, the resonances of residues $4-6$ and $9-10$ in the $\mathrm{N}$-terminal unstructured region, residues 56-57 in the long DE loop, 91 and 95 at the beginning 
and end of strand F, and 116 and 119-121 in strand $H$ were not observed in the apo-TTR spectrum because of fast amide hydrogen exchange with the solvent. At $\mathrm{pH} 7.0$, only residues 56-57 were still not detected. The assignment of TTR resonances when bound to each of the two prototypic ligands, respectively, the monovalent tafamidis ${ }^{4}$ and the bivalent mds84, ${ }^{7}$ were confirmed by $3 \mathrm{D}\left[{ }^{1} \mathrm{H},{ }^{13} \mathrm{C},{ }^{15} \mathrm{~N}\right]$ TROSY HNCA. Based on these assignments, we have compared the respective holo-TTR forms with apo-TTR.

According to the $K_{\mathrm{d}}$ values of 2 and $200 \mathrm{nM}$ reported for the first and second sites occupied in the TTR-tafamidis complex, ${ }^{10}$ at a protein concentration of $50 \mu \mathrm{M}$ and 1 equiv of tafamidis, $91 \%$ of TTR molecules have a single ligand bound and $4 \%$ have two ligands bound. The NMR resonances will thus be dominated by the singly bound form (but note this gives rise to two different signals, corresponding to the different chemical environments in the two-halves of the TTR tetramer). In the presence of 2 equiv of tafamidis, $8 \%$ have a single ligand bound and $92 \%$ have two ligands bound, producing spectra dominated by the fully bound form. The avid pseudo-irreversible binding of mds 84 and the simultaneous occupancy of the two binding sites ${ }^{7}$ allows the complete saturation of TTR by a single equivalent of the bivalent ligand. Based on these known stoichiometric states, NMR can highlight the structural modifications caused by the occupancy of one or both sites by the monovalent ligand and both sites by the bivalent ligand.

The appearance of NMR resonances during titration with a ligand is determined by the exchange rate, $k_{\mathrm{ex}}$, relative to the frequency difference, $\Delta \omega$, between the free and bound state. When chemical shift differences are large $\left(\Delta \omega \gg k_{\mathrm{ex}}\right)$, the exchange is slow so that free and bound peaks are observed simultaneously. Alternatively, if chemical shift differences are small $\left(\Delta \omega \ll k_{\mathrm{ex}}\right)$, then the resonance will be in the fast exchange regime and the observed resonance position is the population-weighted average of the free and bound chemical shift. ${ }^{20}$ Moreover, changes in NMR line shapes across titrations can also be highly sensitive to microscopic reaction mechanisms. $^{21,22}$

The mechanism of the peculiar negative cooperativity of binding of TTR to thyroxine, tafamidis and most other monovalent ligands, can be explored by NMR. At first glance, inspection of $2 \mathrm{D}\left[{ }^{1} \mathrm{H},{ }^{15} \mathrm{~N}\right]$-TROSY spectra acquired at varying ligand concentrations showed the simultaneous presence of peaks of the free and bound form (Figure S2), consistent with slow exchange on the chemical shift timescale both for tafamidis and mds84, but more thorough analysis revealed more complex exchange behavior. The numerous peaks that shift in the holoform are clearly observable for tafamidis and mds 84 in Figure $1 \mathrm{E}, \mathrm{F}$.

Figure 2 shows magnified views of the behavior of two representative TTR residues, E72 and $\mathrm{H} 88$, across the titration with mds84. For E72 (Figure 2A), which is representative of the majority of TTR resonances, mds 84 induced a progressive decrease of the peak intensity of the apo-form and an increase of the same peak intensity in the holo-form, that is, slow exchange. For a group of 14 residues (Figure 2B), exemplified by H88, the corresponding peaks were not observed in the holo-TTR, indicating the presence of either intermediate exchange or micro second-millisecond dynamics within the bound state. The chemical shift perturbation (CSP) values between the apo and holo form are reported in Figure 2C and Movie M1.

A titration of TTR with tafamidis disclosed a more complex NMR chemical exchange behavior. This binding can be represented by three different classes of peaks exemplified by D99, S117, and L55 (Figure 3). According to the observed chemical shift changes, two phases can be distinguished: the first, during the occupancy of the first binding site is followed by a second phase when both sites are saturated. In the first phase, two of the four TTR subunits are directly involved in the tafamidis binding (bound subunits) while the remaining two (unbound subunits) are not.

During titration from a tafamidis/TTR ratio of $0: 1$ to $1: 1$, shown in Figure 3A, the initial D99 peak corresponding to unbound TTR subunits progressively decreased, while the peak corresponding to bound subunits increased, similar to the slow exchange behavior of E72 during addition of mds84. In contrast, the initial unbound $\mathrm{S} 117$ resonance decreased and broadened prior to the observations of resonances from bound subunits (Figure 3B). Additionally, for a subset of residues including L55, progressive chemical shift changes and a decrease in intensity were observed from the initial unbound resonance up to a 1:1 ratio (Figure 3C, red arrows). The resonance at this point corresponds to unoccupied subunits within singly-bound TTR tetramers, and the observed changes indicate both that a component of the binding process occurs within the fast exchange regime, and that the conformation of these subunits is altered relative to the apo state. Simultaneously, a second peak was observed, increasing in intensity and corresponding to bound subunits within occupied TTR tetramers. A small change in its chemical shift was observed between 1 and 2 equiv, also indicating a change in the conformation of subunits between singly and fully occupied TTR tetramers. For all the three classes of peaks, in the second phase of the reaction, the apo-form vanished and the holo-form reached its maximum intensity. Figure 3D,E and Movies M2 and M3 summarize the conformational changes occurring in the first and second phase, respectively. This complex behavior cannot be obtained from a simple two-state binding equilibrium. Instead, the simplest model consistent with the observed NMR lineshapes ${ }^{21}$ is that the first equivalent of tafamidis binds through a scheme where the initial binding occurs in fast exchange, followed by a slower intramolecular rearrangement. A second molecule of tafamidis may then bind to this singly-bound state, although it is not clear from our data whether this occurs in a simple two-state manner or again via a more complex multistep mechanism.

At a 1:1 ratio, the residues involved in the fast formation of the complex and those involved in the final rearrangement of the complex are shown in Figure 3D. The residues within the binding site showing a chemical shift change were K15, L17 within strand A and R103 and R104, located just before and at the N-terminus of strand G. Other residues involved, but located outside of the binding site, were A25 and I26 in the A'B loop, S52 in the CD loop, and H90 and V94 in strand F. The chemical shift variations of the peaks in the rearranged complex outnumbered those of the initially formed complex and were mostly located in the dimer interface and in particular throughout strand $\mathrm{A}, \mathrm{A}^{\prime}$, at $\mathrm{A} 120$ and V122 of strand $\mathrm{H}$, strand $\mathrm{C}$, and the $\mathrm{C}$-term of strand $\mathrm{G}$, localizing the site of interaction with tafamidis. However, variations also propagated to the outer strand F (H90 and V94) to the helix with the NH and the indole side chain of W79 where conformational changes may be hypothesized.

Surface Plasmon Resonance Analysis Reveals Two Different Binding Kinetics of Tafamidis by TTR. The NMR-based evidence describes, for the first time, that a structural rearrangement occurs in TTR upon occupancy of 
A

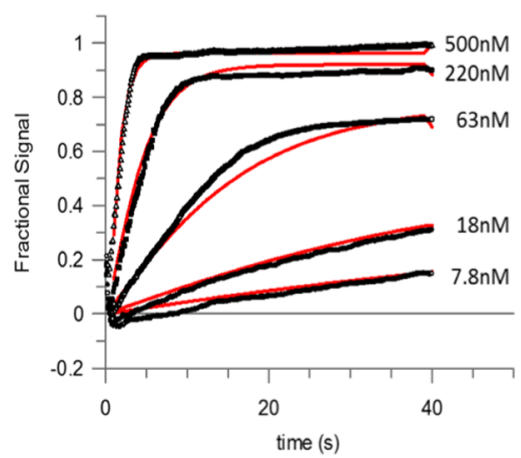

B

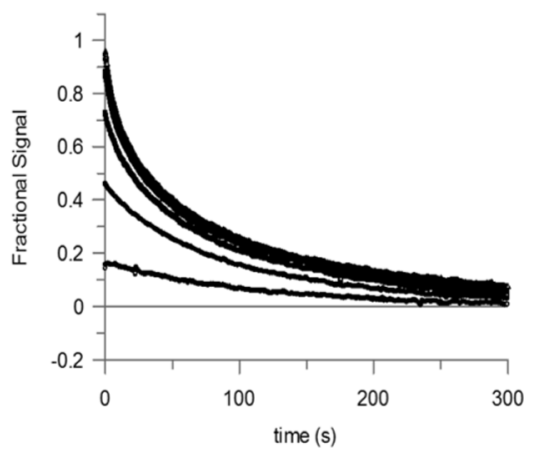

Figure 4. SPR analysis of ligand binding by TTR. (A,B) Association and dissociation curves derived by titration of immobilized TTR with tafamidis. The black symbols show experimental measurements and red lines are global fitting of the results, as described in the Experimental Section.

the first site. We used surface plasmon resonance (SPR) to analyze the TTR tafamidis interaction in order to measure the contribution of $k_{\mathrm{on}}$ and $k_{\text {off }}$ to the binding dynamics that produce the observed negative cooperativity. Global fitting of the data from the association and dissociation phases (Figure 4) provided estimates of the on-rate $\left(k_{1}\right.$ and $\left.k_{3}\right)$ and off-rate $\left(k_{2}\right.$ and $k_{4}$ ) (Table 1$)$ for Scheme 1 described in the Experimental Section.

Table 1. Association and Dissociation Parameters for the Binding of Tafamidis to $\operatorname{TTR}^{a}$

$\begin{array}{ll}k_{1} & (4.5 \pm 2.2) \times 10^{6} \mathrm{M}^{-1} \mathrm{~s}^{-1} \\ k_{2} & (0.008 \pm 0.001) \mathrm{s}^{-1} \\ k_{3} & (3.5 \pm 1.0) \times 10^{6} \mathrm{M}^{-1} \mathrm{~s}^{-1} \\ k_{4} & (0.060 \pm 0.006) \mathrm{s}^{-1} \\ K_{\mathrm{d} 1} & (3 \pm 1) \times 10^{-9} \mathrm{M} \\ K_{\mathrm{d} 2} & (22 \pm 7) \times 10^{-9} \mathrm{M}\end{array}$

${ }^{a}$ Kinetics and thermodynamics constants are reported with their standard deviations. The $K_{\mathrm{d} 1}$ and $K_{\mathrm{d} 2}$ are reported as the averages of the ratios of $k_{2} / k_{1}$ and $k_{4} / k_{3}$, respectively, of three independent experiments.

The calculated association rate constants were comparable for both sites in three independent experiments. However, the dissociation rate constants were distinctly different. The rate at the second site was an order of magnitude faster than at the first site, indicating that the primary driver of the observed negative cooperativity may be a faster dissociation from the second binding site than from the first. The NMR slow exchange phase is in full agreement with the off-rates determined by SPR and represents the SPR rate-determining step, as, in contrast to NMR, SPR cannot detect structural variations. The following comparison considers that while NMR exchange regimes are determined by the exchange rate, $k_{\mathrm{ex}}=k_{\mathrm{on}}[\mathrm{L}]+k_{\text {off }}$, relative to the frequency difference between states, and are therefore technically dependent on the free ligand concentration, at 50\% occupancy, where the appearance of the spectrum is most sensitive to chemical exchange, $k_{\text {ex }}$ is simply equal to twice the off rate. The off-rate, $k_{2}=0.008 \mathrm{~s}^{-1}$, for occupation of a single binding site, can be compared to the chemical shift difference calculated between the apo-form and the TTR-tafamidis complex at $1: 1$ stoichiometry. It resulted in an average value $\Delta \omega^{1 \mathrm{H}}(\mathrm{rad} / \mathrm{s})=511.5(\min =90.4, \max =2281.0)$ and $\Delta \omega^{15 \mathrm{~N}}$ $(\mathrm{rad} / \mathrm{s})=242.0(\mathrm{~min}=62.7, \max =891.6)$. Similarly, the offrate, $k_{4}=0.06 \mathrm{~s}^{-1}$, for the occupation of the second binding site compares to the chemical shift difference between holo-TTR and TTR with a single binding site occupied, where the average values were $\Delta \omega^{1 \mathrm{H}}(\mathrm{rad} / \mathrm{s})=465.6(\min =175.9, \max =1286.8)$ and $\Delta \omega^{15 \mathrm{~N}}(\mathrm{rad} / \mathrm{s})=203.8(\min =63.2, \max =874.8)$. Both offrates were much smaller than the $\Delta \omega$ for ${ }^{1} \mathrm{H}$ and ${ }^{15} \mathrm{~N}$ nuclei, confirming a slow exchange on the chemical shift timescale.

Comparative Analysis of Saturation Binding of Different Ligands Reveals Global Protein Structural Effects on TTR. An in-depth analysis of the NMR spectra at complete saturation with both ligands was carried out. The numerous CSPs observed in holo-TTR with tafamidis and mds84, respectively, are shown in Figure 5, and highlighted in blue in Figure 6.

Both ligands produced a marked perturbation at the dimerdimer interface (strands DAGH- $\mathrm{H}^{\prime} \mathrm{G}^{\prime} \mathrm{A}^{\prime} \mathrm{D}^{\prime}$; Figure 6A,B upper panel) in regions corresponding to the three halogen binding pockets (HBPs) originally described by Blake \& Oatley ${ }^{3}$ and then refined by Palaninathan. ${ }^{13}$ HBP 1 is the wide outer pocket formed by M13, K15, L17, T106, A108, V121, and HBP2 comprises L17, A108, A109, L110, and the inner pocket, HBP3, is composed of A108, L110, S117, and T119. Other effects specific for tafamidis or mds 84 were also detected. In particular, the full occupancy of the two sites affected all the observed residues of the HBPs HBP3-HBP1. Moreover, V121, not observable in the apo-form, became visible with tafamidis only. CSPs were also detected in strands $\mathrm{B}, \mathrm{E}$, and $\mathrm{F}$ of the outer $\beta$ sheet (Figure 6A,B middle panel), as well as loops $\mathrm{AB}, \mathrm{BC}$, only with tafamidis, and $\mathrm{CD}, \mathrm{DE}, \mathrm{EH} 1$, only with mds84, and $\mathrm{H} 1$ helix. These surprisingly significant remote perturbations in the BEF strands and in the helix, the outer part of the molecule distant by about 12-36 $\AA$ from the ligand binding pocket (Figure 6A,B middle panel), were not predicted, according to the HBP model, to change their conformation upon binding, in contrast to the chemical shift deviations expected for the actual binding residues.

The residues not belonging to HBPs and shifting more than 1 standard deviation from the mean were all in loop AB; $19-21$ with bound tafamidis and 18,20 , and 25 with mds84. The binding of mds 84 caused the greatest CSP in residue A25, which makes a H-bond with $\mathrm{T}_{4} 9 \mathrm{O}_{\gamma 1}$ (Figure $6 \mathrm{~B}$ lower panel), suggesting that strengthening of this $\mathrm{H}$-bond may be responsible for inhibition of the amyloidogenic cleavage of the residue 4849 peptide bond by serine proteases. We note that loop $A B$ is central to the weak dimer-dimer association, as it interacts with the opposite loop GH of a different chain that was also perturbed upon binding. The bivalent ligand, mds84, was particularly 
A
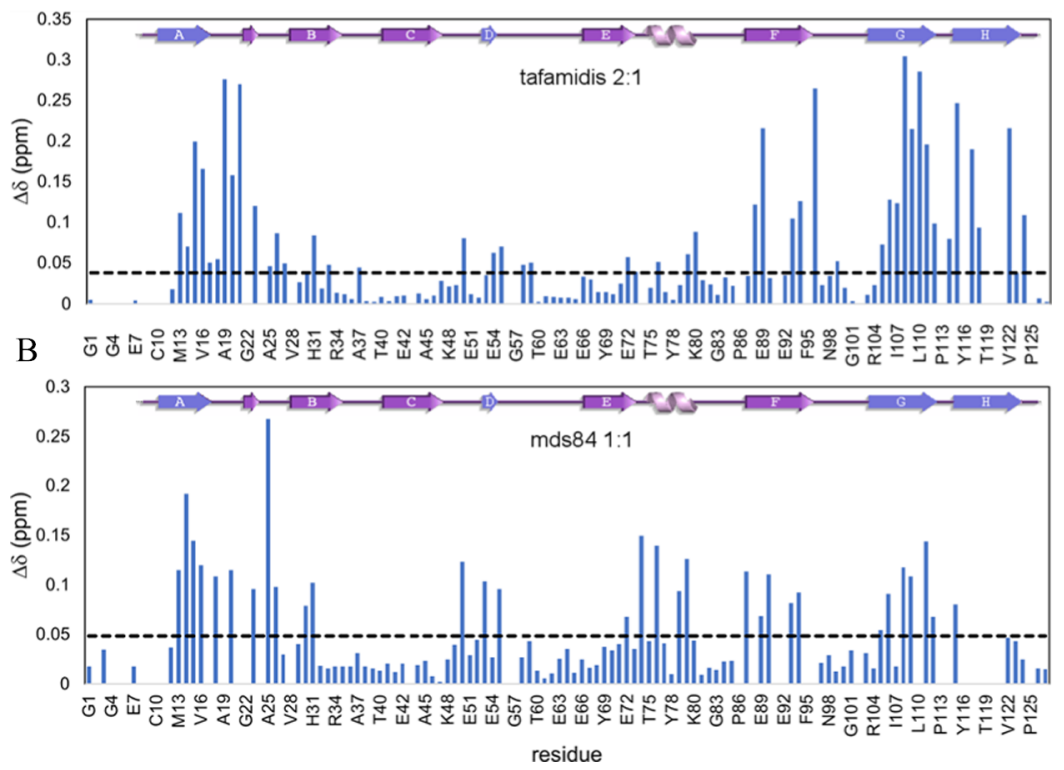

Figure 5. Observed CSPs of TTR in the presence of tafamidis and mds84. (A) Combined amide ${ }^{1} \mathrm{H}$ and ${ }^{15} \mathrm{~N}$ chemical shift for holo-TTR with tafamidis at 2:1 and (B) in the presence of mds84 at a 1:1 ratio. The dashed line shows the mean chemical shift variation after the removal of outliers.

effective in perturbing loop $\mathrm{CD}$, strands $\mathrm{D}$ and $\mathrm{E}$ and helix $\mathrm{H} 1$. Binding of either ligand had major effects on strand F, with residues E89 and T96 shifted more than 3 standard deviations from the mean in the presence of tafamidis. Their side chains participate in an interchain hydrogen bond, also involving residue $\mathrm{K} 76$, to form a network connecting the apices of two monomers. In the presence of mds84, the major shifts were in strand F residues $\mathrm{H} 90$ and V93 (Figure 6A lower panel).

Interestingly, a few peaks (in red in Figure 6) became visible only when the ligands occupied the HBPs: K9 and A120 appeared with both tafamidis and mds8, whereas A91 and V121 were observed only in the presence of the monovalent ligand (Figure S3). The appearance of A120 and V121, located in strand $\mathrm{H}$, could be due to a slower exchange rate of the amide with water than in the apo-form. This may reflect the stabilization of the interchain hydrogen bonds between A120 (chain A, C) and Y114 of strand $\mathrm{H}^{\prime}$ (chain B, D) and the intraprotomer hydrogen bond between strand $\mathrm{G}$ residues $\mathrm{V} 121$ and T106, confirmed by a temperature coefficient for the amide hydrogen of A120 (see Table S1) higher than $-4.5 \mathrm{ppb} / \mathrm{K}^{23}$ Interestingly, the amide of L55, exhibiting a small chemical shift change both with tafamidis and mds84, became $\mathrm{H}$-bonded in the holo-form (Table S1). Its structural location, in front of K48, is consistent with the ligand-mediated reduced susceptibility to proteolysis of the CD loop.

\section{DISCUSSION AND CONCLUSIONS}

We report here two key molecular structural aspects of ligand binding by TTR, elucidated by NMR analysis:

1 We have shown that an intermediate conformational state forms when TTR binds a monovalent ligand in only one of its two identical binding sites;

2 We have discovered long distance (12-36 ̊) structural effects on the protein when the binding sites are occupied by ligands and we have shown that the structural effects are more extensive with a bivalent than a monovalent ligand.
Negative cooperativity of binding of thyroxine, the natural TTR ligand, first described in $1975,{ }^{24}$ has been attributed to putative subtle differences between the two binding sites. ${ }^{25-28}$ This poorly understood aspect of negative cooperativity is nevertheless important because it affects the possibility to fully saturate plasma TTR with stabilizing ligand compounds to most effectively protect against TTR amyloid fibril formation. ${ }^{10}$ Although the $2 \mathrm{D}\left[{ }^{1} \mathrm{H},{ }^{15} \mathrm{~N}\right]$ TROSY spectra do not reveal any asymmetry of apo-TTR, we have shown here that occupancy of the first site by tafamidis is followed by a rearrangement of the binding site, involving not only residues located at the dimer interface but also residues in the outer TTR surface and not in contact with the ligand. The scheme in Figure 7A summarizes what emerges from our current study of an unusually complex equilibrium pattern. Our results clearly show an induced fit mechanism in which the initial fast occupancy of the first binding site is followed by a slower rearrangement of the site enabling a tighter embrace of the monovalent ligand. Moreover, the geometry of the binding pocket is altered when two ligands, rather than one, are bound. The simpler equilibrium pattern in the case of the binding of mds84 is described in Figure 7B.

This NMR derived model is supported by direct SPR measurements of the binding dynamics, in which the 7 -fold difference in the observed affinities at the two sites is driven by different off-rates ( $k_{2}$ and $k_{4}$, Table 1$)$. The calculated affinity of the second site based on the SPR data is higher than previously reported $^{4}(22 \mathrm{nM}$ vs $200 \mathrm{nM})$ and the reasons of this are not clear. One possibility is that immobilization of the tetramer to the SPR surface impacts on the dynamics of the conformational changes observed in solution, and limiting the change in off rate induced by binding at the first site.

Our NMR findings with full occupancy of TTR by tafamidis or mds 84 show that substantial perturbations are not confined to the proximity of ligands but are also propagated into the outer region, comprising the BCEF $\beta$-sheet and the helix. Aromatic rings, present in tafamidis and mds84, are well known to affect the chemical shift of neighboring amides, but this effect decreases with the third power of the distance from the center 


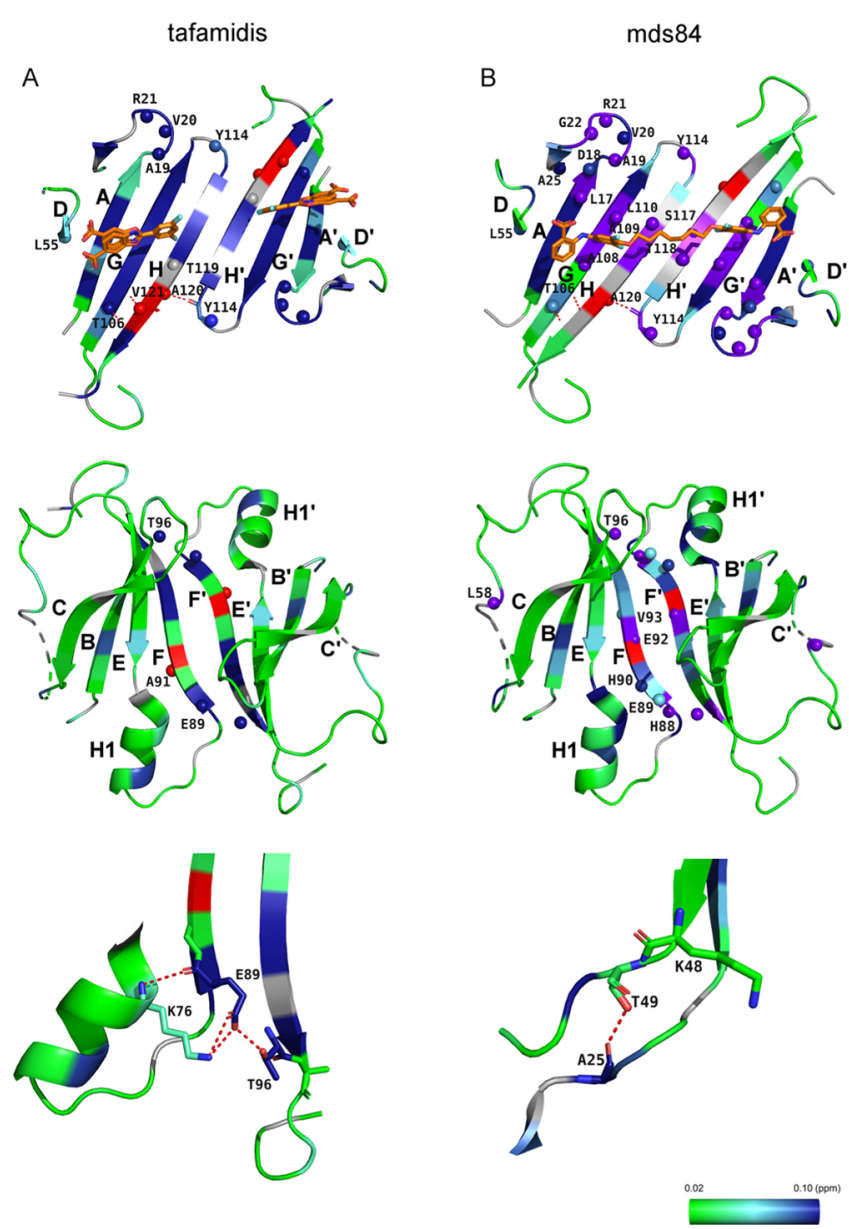

Figure 6. Structural effects of bound tafamidis and mds84 at 2:1 and 1:1 molar ratios to TTR, respectively. (A) Effects of tafamidis on residues within the protein central $\beta$-sheet (upper panel) and in its external surface (middle panel). The lower panel illustrates a network of $\mathrm{H}$ bonds between K76, E89 of chain A and T96 of chain B. (B) Effects of mds8 4 on residues within the protein central $\beta$-sheet (upper panel) and in its external surface (middle panel). The lower panel illustrates the $\mathrm{H}$ bond between A25 and T49. A green-light blue-blue gradient depicts the extent of chemical shift change and those that become visible only once the ligand is bound are shown in red. Purple spheres represent amides that disappear only upon binding. When no information is available, the residues are shown in gray. The PDB codes are 3TCT for the TTR complex with tafamidis and 3IPE with mds84.

of the ring ${ }^{20}$ and cannot cause the distant CSPs we report here. A radius $12 \AA$ sphere centered on the ligand rings defines a region, external to the spheres, where any perturbation can only be attributed to allosteric structural changes induced by ligand binding. Furthermore, significant stabilization by additional hydrogen bonding, as witnessed by temperature coefficients measurements, can be envisaged for residues L55, A120, and V121. L55 is located opposite to K48; A120 is involved in the intermolecular pairing between strands $\mathrm{H}$ and $\mathrm{H}^{\prime}$; and V121 forms an intramolecular H-bond with T106 of the facing strand G. Evidence from ${ }^{19} \mathrm{~F}$ NMR also suggests that A120, together with F87, may additionally be critical for TTR stabilization as shown by the A120L TTR variant favoring a mis-packed state. ${ }^{17}$

Our data show that the binding of the monovalent and the bivalent ligands affects protein dynamics differentially. The disappearance of amides within the dimer interface from 2D TROSY maps when mds 84 is bound is attributable to protein dynamics in the micro second-millisecond time scale,

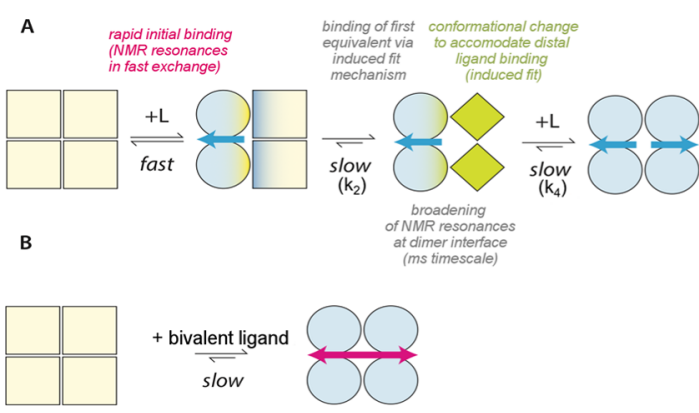

Figure 7. Scheme of the binding of tafamidis and mds 84 by TTR. (A) Binding of tafamidis. Apo-TTR, which is a symmetrical homotetramer is shown as pale-yellow squares. Binding of a single molecule of tafamidis initially induces changes in the occupied half molecule dimer, shown here as light-blue green circles, and is then followed by conformational changes in the unoccupied dimer, shown as green diamonds. When the second tafamidis molecule is bound, the TTR tetramer becomes symmetrical again, with the protomers illustrated as light blue circles. The off-rates measured by SPR, $k_{2}=0.008 \mathrm{~Hz}$ and $k_{4}=$ $0.06 \mathrm{~Hz}$, are consistent with global slow exchange in which the slower $k_{4}$ is responsible for the negative cooperative effect of binding of this monovalent ligand. (B) Binding of mds 84 induces symmetrical changes into the holo-TTR shown as light blue circles.

unfavorable for NMR observation. Residues in loops $A B, D E$, $\mathrm{H} 1 \mathrm{~F}$, and strand $\mathrm{F}$ are similarly affected, probably reflecting a change in mobility due to the occupancy of the central part of the TTR channel by the linker chain between the two head groups of mds84. Neutron scattering studies of H/D exchange in the most highly amyloidogenic TTR variant, S52P, show, counterintuitively, greater exchange when tafamidis is bound than in the TTR protein alone. ${ }^{29}$ Ligand binding by TTR evidently can increase accessibility/mobility and normal mode analysis simulations have previously shown low frequency subunit motion in the apo-protein that can be modified in the holo-form. ${ }^{27}$ Remarkably, residue A25, which is not within the HBPs, shows the largest NMR chemical shift change in the mds84 complex and just above the mean with bound tafamidis. The carbonyl of A25 is hydrogen bonded to the side chain of T49 and the stronger observed intensity of the A25 peak shift is consistent with our experimental observation that mds84 confers the highest protection against the proteolytic cleavage of the peptide bond 48-49. ${ }^{10}$ As previously highlighted by Mangione et al., ${ }^{8}$ conformational changes favoring stability of the cluster of $\mathrm{H}$-bonds involving residues S50, S52, and E54, that are partially disrupted in the highly amyloidogenic variant S52P, should protect against this amyloidogenic cleavage. The observed CSPs of S50, G53, E54, and L55, together with the $\mathrm{H}$-bond of the latter when the HBPs are occupied, are therefore interpretable as decreased accessibility to proteolysis of the K48-T49 bond.

The changes reported here in solution have not previously been detected in a plethora of X-ray structures of TTR. As discussed extensively by Palaninathan, ${ }^{13}$ the large number of reported X-ray crystal structures, including wild type TTR, numerous TTR variants and TTR complexed with a variety of different ligands, do not show any structural alterations that could account for their different propensity to unfold and then aggregate into amyloid fibrils. In particular, a detailed analysis of the pairwise superimposition of the X-ray structure of TTR complexed with tafamidis (3TCT) ${ }^{4}$ or mds84 (3IPE) ${ }^{7}$ with the structure of apo-TTR (5CN3) gives $\mathrm{C}_{\alpha} \mathrm{rmsd}$ values of 0.618 and 
$0.315 \AA$, respectively, accounted for by a small deviation in the FG loop (residues 100-103).

In conclusion, our present NMR observations of free TTR alone compared to TTR complexes with different ligands, have revealed conformational changes never previously seen with other approaches including high-resolution X-ray crystallography. The direct NMR structural evidence of long distance conformational changes induced by ligands is consistent with the indirect evidence of long distance effects provided by experimental observations of the inhibitory effects of ligand binding on limited proteolysis of TTR and its amyloidogenesis. In the future, NMR signatures of the effects of ligand binding could make a valuable contribution to design and development of novel ligands for use as inhibitors of TTR amyloid fibril formation.

\section{EXPERIMENTAL SECTION}

Ligand Preparation. Tafamidis was synthesized by Selcia Ltd, Ongar, Essex UK. Analysis was performed in accordance with Internal quality program procedures by the Analytical Support Group, Selcia. Identity of the test item is confirmed by NMR and LCMS analysis. Chemical purity is determined by ${ }^{1} \mathrm{H}$ NMR and HPLC-UV analysis. Chemical purity was assessed as $94 \%$ (relative area by NMR); of the $6 \%$ impurities, $2.3 \%$ is attributable to methanol. Chemical purity is $98.4 \%$ (\% AUC).

Mds84 was synthesized by WuXi AppTec, Shanghai, China. The sample was $>99 \%$ pure by HPLC-UV analysis. ${ }^{1} \mathrm{H}$ NMR analysis is consistent with the purity.

NMR Spectroscopy. NMR spectra were acquired at $310 \mathrm{~K}$ with a Bruker AVANCE 700 and $800 \mathrm{MHz}$ NMR spectrometer on a $\mathrm{U}_{-}^{2} \mathrm{H}$, ${ }^{13} \mathrm{C},{ }^{15} \mathrm{~N}$-labeled recombinant TTR samples at concentrations of 50-90 $\mu \mathrm{M}$ in PBS buffer at $\mathrm{pH} 7.0$ and 7.4 in a mixture $\mathrm{H}_{2} \mathrm{O} / \mathrm{D}_{2} \mathrm{O} 90 / 10 \%$.

The addition of 4,4-dimethyl-4-silapentane-1-sulfonic acid (DSS) for reference purposes led to the observation that DSS binds to the TTR HBP (see Figure S4).

Ligand stock solutions at $10 \mathrm{mM}$ were prepared in DMSO- $d_{6}$ and additions up to $6 \mu \mathrm{L}$ into $600 \mu \mathrm{L}$ of $50 \mu \mathrm{M}$ protein solution were made. The effect of DMSO- $d_{6}$ at up to $1 \% \mathrm{v} / \mathrm{v}$ on chemical shift was negligible (see Figure S5). 2D $\left[{ }^{1} \mathrm{H},{ }^{15} \mathrm{~N}\right]$ TROSY spectra were acquired at ligand/ protein ratios of $0,0.5,1.0,1.5$, and 2.0 with tafamidis, the monovalent ligand, and at $0,0.5$, and 1.0 with mds 84 , the bivalent ligand.

3D HNCA spectra were also recorded in the presence of the ligands to confirm the assignment.

To express changes in the chemical shifts of the individual amide pairs, a combined chemical shift change (in ppm) was defined ${ }^{30}$ as

$$
\Delta \delta=\sqrt{\left(\Delta \delta_{\mathrm{HN}}\right)^{2}+\left(\frac{\Delta \delta_{\mathrm{N}}}{6.5}\right)^{2}}
$$

The involvement of amide hydrogens in $\mathrm{H}$-bonds was estimated by a series of $112 \mathrm{D}$ TROSY $\left[{ }^{1} \mathrm{H},{ }^{15} \mathrm{~N}\right]$ spectra acquired at temperature varying from 289 to $313 \mathrm{~K}$ for apo-TTR, for holo-TTR with tafamidis and mds84 in a 2:1 and 1:1 ligand/protein ratio, respectively.

Surface Plasmon Resonance. The binding of tafamidis to TTR was monitored using SPR performed using a Biacore T200 instrument (GE Healthcare) with streptavidin coated sensor chips.

Data were fitted to the model described by Scheme 1 . The measured fractional response is then given by $\left\{[A B+(2 A B B)] / A_{i}\right\}$ where $A_{i}$ is the notional concentration of TTR on the surface. Rate parameters were determined from the global fitting of the association data to the binding equilibria as defined by Scheme 1 . The dissociation rates $\left(k_{2}\right.$ and $\left.k_{4}\right)$ were separately derived from the fitting of the dissociation data to a

\section{Scheme 1}

double exponential equation with $k_{2}$ and $k_{4}$ being allocated to the first and second binding steps, respectively, based on the reduced contribution of a second exponential at the lower concentrations of tafamidis, and the global fit repeated to determine the on rate constants with the dissociation constants fixed. The affinity of tafamidis at each of the two binding sites was determined from the ratio of rate constants, $K_{\mathrm{d} 1}=k_{2} / k_{1}$ and $K_{\mathrm{d} 2}=k_{4} / k_{3}$, respectively.

Other Methods. Recombinant TTR production, details on NMR spectra acquisition, and on SPR data acquisition are described in Supporting Information Methods.

\section{ASSOCIATED CONTENT}

\section{Supporting Information}

The Supporting Information is available free of charge on the ACS Publications website at DOI: 10.1021/acs.jmedchem.9b01037.

Molecular formula strings (CSV)

Methods of TTR expression and purification, NMR spectra acquisition and on SPR data acquisition; additional table of NMR temperature coefficients of apo and holo TTR with tafamidis and mds84 (Table S1); and figures reporting TTR dimer structure, 2D TROSY spectra at sub-stoichiometric ligand/TTR ratios showing the presence of free and bound peaks, NMR peak intensities, DSS, and DMSO effects (PDF)

Structural effects of mds84 and tafamidis (MPG)

Structural effects of mds84 and tafamidis (MPG)

Structural effects of mds84 and tafamidis (MPG)

\section{AUTHOR INFORMATION}

\section{Corresponding Authors}

*E-mail: alessandra.corazza@uniud.it (A.C.).

*E-mail: v.bellotti@ucl.ac.uk (V.B.).

ORCID $\odot$

Alessandra Corazza: 0000-0003-2272-1928

Christopher A. Waudby: 0000-0001-7810-3753

Author Contributions

The study was conceived, designed and supervised by V.B. and A.C. A.C., G.V., C.A.W., P.P.M., D.C., P.N. and G.W.T. performed research. A.C., G.V., C.A.W., J.C. analysed and interpreted NMR data. R.B. and I.U. performed and analysed Biacore data. J.C. and M.B.P. contributed to discussion. All the authors analysed and interpreted the data. The paper was written by V.B., A.C. and M.B.P. and reviewed and approved by all coauthors.

Notes

The authors declare no competing financial interest.

\section{ACKNOWLEDGMENTS}

This work was supported by investment from the University College London Technology Fund and grants from the U.K. Medical Research Council (MR/K000187/1 and MR/ R016984/1), the Rosetrees Trust/Royal Free Charity PhD programme (M427), the Cariplo Foundation (projects 20140700), the Italian Ministry of Health (Ricerca Finalizzata RF 2013 02355259) and the Istituto Nazionale di Biostrutture e Biosistemi. Core support for the Wolfson Drug Discovery Unit is provided by the UK National Institute for Health Research Biomedical Research Centre and Unit Funding Scheme via the UCLH/UCL Biomedical Research Centre and by the UCL Amyloidosis Research Fund. We acknowledge the use of the UCL NMR Centre and the MRC Biomedical NMR Centre at 
the Francis Crick Institute, London, and thank the staff for their support. We acknowledge MIUR Dipartimento di Eccellenza UniPV. We also thank Professor Trevor Forsyth for kindly providing the plasmid used for recombinant TTR production.

\section{ABBREVIATIONS}

TTR, transthyretin; rmsd, root mean square deviation; TROSY, transverse relaxation-optimized spectroscopy; CSP, chemical shift perturbation; SPR, surface plasmon resonance; HBP, halogen binding pocket; DSS, 4,4-dimethyl-4-silapentane-1sulfonic acid

\section{REFERENCES}

(1) Rowczenio, D. M.; Noor, I.; Gillmore, J. D.; Lachmann, H. J.; Whelan, C.; Hawkins, P. N.; Obici, L.; Westermark, P.; Grateau, G.; Wechalekar, A. D. Online registry for mutations in hereditary amyloidosis including nomenclature recommendations. Hum. Mutat.. 2014, 35-E2403-E2412. DOI: 10.1002/humu.22619

(2) Hawkins, P. N.; Ando, Y.; Dispenzeri, A.; Gonzalez-Duarte, A.; Adams, D.; Suhr, O. B. Evolving landscape in the management of transthyretin amyloidosis. Ann. Med. 2015, 47, 625-638.

(3) Blake, C. C. F.; Oatley, S. J. Protein-DNA and protein-hormone interactions in prealbumin: a model of the thyroid hormone nuclear receptor? Nature 1977, 268, 115-120.

(4) Bulawa, C. E.; Connelly, S.; Devit, M.; Wang, L.; Weigel, C.; Fleming, J. A.; Packman, J.; Powers, E. T.; Wiseman, R. L.; Foss, T. R.; Wilson, I. A.; Kelly, J. W.; Labaudiniere, R. Tafamidis, a potent and selective transthyretin kinetic stabilizer that inhibits the amyloid cascade. Proc. Natl. Acad. Sci. U.S.A. 2012, 109, 9629-9634.

(5) Kelly, J.; Colon, W.; Lai, Z.; Lashuel, H.; McCulloch, J.; McCutchen, S.; Miroy, G.; Peterson, S. Transthyretin quaternary and tertiary structural changes facilitate misassembly into amyloid. Adv. Protein Chem. 1997, 50, 161-181.

(6) Connelly, S.; Choi, S.; Johnson, S. M.; Kelly, J. W.; Wilson, I. A. Structure-based design of kinetic stabilizers that ameliorate the transthyretin amyloidoses. Curr. Opin. Struct. Biol. 2010, 20, 54-62.

(7) Kolstoe, S. E.; Mangione, P. P.; Bellotti, V.; Taylor, G. W.; Tennent, G. A.; Deroo, S.; Morrison, A. J.; Cobb, A. J. A.; Coyne, A.; McCammon, M. G.; Warner, T. D.; Mitchell, J.; Gill, R.; Smith, M. D.; Ley, S. V.; Robinson, C. V.; Wood, S. P.; Pepys, M. B. Trapping of palindromic ligands within native transthyretin prevents amyloid formation. Proc. Natl. Acad. Sci. U.S.A. 2010, 107, 20483-20488.

(8) Mangione, P. P.; Porcari, R.; Gillmore, J. D.; Pucci, P.; Monti, M.; Porcari, M.; Giorgetti, S.; Marchese, L.; Raimondi, S.; Serpell, L. C.; Chen, W.; Relini, A.; Marcoux, J.; Clatworthy, I. R.; Taylor, G. W.; Tennent, G. A.; Robinson, C. V.; Hawkins, P. N.; Stoppini, M.; Wood, S. P.; Pepys, M. B.; Bellotti, V. Proteolytic cleavage of Ser52Pro variant transthyretin triggers its amyloid fibrillogenesis. Proc. Natl. Acad. Sci. U.S.A. 2014, 111, 1539-1544.

(9) Marcoux, J.; Mangione, P. P.; Porcari, R.; Degiacomi, M. T.; Verona, G.; Taylor, G. W.; Giorgetti, S.; Raimondi, S.; SanglierCianférani, S.; Benesch, J. L.; Cecconi, C.; Naqvi, M. M.; Gillmore, J. D.; Hawkins, P. N.; Stoppini, M.; Robinson, C. V.; Pepys, M. B.; Bellotti, V. A novel mechano-enzymatic cleavage mechanism underlies transthyretin amyloidogenesis. EMBO Mol. Med. 2015, 7, 1337-1349.

(10) Verona, G.; Mangione, P. P.; Raimondi, S.; Giorgetti, S.; Faravelli, G.; Porcari, R.; Corazza, A.; Gillmore, J. D.; Hawkins, P. N.; Pepys, M. B.; Taylor, G. W.; Bellotti, V. Inhibition of the mechanoenzymatic amyloidogenesis of transthyretin: role of ligand affinity, binding cooperativity and occupancy of the inner channel. Sci. Rep. 2017, 7, 182 .

(11) Mangione, P. P.; Verona, G.; Corazza, A.; Marcoux, J.; Canetti, D.; Giorgetti, S.; Raimondi, S.; Stoppini, M.; Esposito, M.; Relini, A.; Canale, C.; Valli, M.; Marchese, L.; Faravelli, G.; Obici, L.; Hawkins, P. N.; Taylor, G. W.; Gillmore, J. D.; Pepys, M. B.; Bellotti, V. Plasminogen activation triggers transthyretin amyloidogenesis in vitro. J. Biol. Chem. 2018, 293, 14192-14199.
(12) Johnson, S. M.; Connelly, S.; Wilson, I. A.; Kelly, J. W. Toward optimization of the second aryl substructure common to transthyretin amyloidogenesis inhibitors using biochemical and structural studies. J. Med. Chem. 2009, 52, 1115-1125.

(13) Palaninathan, S. K. Nearly 200 X-ray crystal structures of transthyretin: what do they tell us about this protein and the design of drugs for TTR amyloidoses? Curr. Med. Chem. 2012, 19, 2324-2342.

(14) Cho, Y.; Baranczak, A.; Helmke, S.; Teruya, S.; Horn, E. M.; Maurer, M. S.; Kelly, J. W. Personalized medicine approach for optimizing the dose of tafamidis to potentially ameliorate wild-type transthyretin amyloidosis (cardiomyopathy). Amyloid 2015, 22, 175180.

(15) Sun, X.; Jaeger, M.; Kelly, J. W.; Dyson, H. J.; Wright, P. E. Mispacking of the Phe87 Side Chain Reduces the Kinetic Stability of Human Transthyretin. Biochemistry 2018, 57, 6919-6922.

(16) Liu, K.; Cho, H. S.; Hoyt, D. W.; Nguyen, T. N.; Olds, P.; Kelly, J. W.; Wemmer, D. E. Deuterium-proton exchange on the native wildtype transthyretin tetramer identifies the stable core of the individual subunits and indicates mobility at the subunit interface. J. Mol. Biol. 2000, 303, 555-565.

(17) Leach, B. I.; Zhang, X.; Kelly, J. W.; Dyson, H. J.; Wright, P. E. NMR Measurements Reveal the Structural Basis of Transthyretin Destabilization by Pathogenic Mutations. Biochemistry 2018, 57, 44214430.

(18) Ikura, M.; Kay, L. E.; Bax, A. A novel approach for sequential assignment of $1 \mathrm{H}, 13 \mathrm{C}$, and $15 \mathrm{~N}$ spectra of proteins: heteronuclear triple-resonance three-dimensional NMR spectroscopy. Application to calmodulin. Biochemistry 1990, 29, 4659-4667.

(19) Pervushin, K.; Riek, R.; Wider, G.; Wuthrich, K. Attenuated T2 relaxation by mutual cancellation of dipole-dipole coupling and chemical shift anisotropy indicates an avenue to NMR structures of very large biological macromolecules in solution. Proc Natl Acad Sci U S A 1997, 94, 12366-12371.

(20) Williamson, M. P. Using chemical shift perturbation to characterise ligand binding. Prog. Nucl. Magn. Reson. Spectrosc. 2013, $73,1-16$.

(21) Kovrigin, E. L. NMR line shapes and multi-state binding equilibria. J. Biomol. NMR 2012, 53, 257-270.

(22) Waudby, C. A.; Ramos, A.; Cabrita, L. D.; Christodoulou, J. TwoDimensional NMR Lineshape Analysis. Sci. Rep. 2016, 6, 24826.

(23) Baxter, N. J.; Williamson, M. P. Temperature dependence of $1 \mathrm{H}$ chemical shifts in proteins. J. Biomol. NMR 1997, 9, 359-369.

(24) Ferguson, R. N.; Edelhoch, H.; Saroff, H. A.; Robbins, J.; Cahnmann, H. J. Negative cooperativity in the binding of thyroxine to human serum prealbumin. Biochemistry 1975, 14, 282-289.

(25) Neumann, P.; Cody, V.; Wojtczak, A. Structural basis of negative cooperativity in transthyretin. Acta Biochim Pol 2001, 48, 867-875.

(26) Tomar, D.; Khan, T.; Singh, R. R.; Mishra, S.; Gupta, S.; Surolia, A.; Salunke, D. M. Crystallographic study of novel transthyretin ligands exhibiting negative-cooperativity between two thyroxine binding sites. PLoS One 2012, 7, No. e43522.

(27) Cianci, M.; Folli, C.; Zonta, F.; Florio, P.; Berni, R.; Zanotti, G. Structural evidence for asymmetric ligand binding to transthyretin. Acta Crystallogr., Sect. D: Biol. Crystallogr. 2015, 71, 1582-1592.

(28) Zanotti, G.; Vallese, F.; Ferrari, A.; Menozzi, I.; Saldaño, T. E.; Berto, P.; Fernandez-Alberti, S.; Berni, R. Structural and dynamics evidence for scaffold asymmetric flexibility of the human transthyretin tetramer. PLoS One 2017, 12, No. e0187716.

(29) Yee, A. W.; Aldeghi, M.; Blakeley, M. P.; Ostermann, A.; Mas, P. J.; Moulin, M.; de Sanctis, D.; Bowler, M. W.; Mueller-Dieckmann, C.; Mitchell, E. P.; Haertlein, M.; de Groot, B. L.; Boeri Erba, E.; Forsyth, V. T. A molecular mechanism for transthyretin amyloidogenesis. Nat. Commun. 2019, 10, 925.

(30) Mulder, F. A. A.; Schipper, D.; Bott, R.; Boelens, R. Altered flexibility in the substrate-binding site of related native and engineered high-alkaline Bacillus subtilisins. J. Mol. Biol. 1999, 292, 111-123. 